\title{
CAT: the Copying Approach to Tabling
}

\author{
Bart Demoen Konstantinos Sagonas \\ Department of Computer Science \\ Katholieke Universiteit Leuven \\ B-3001 Heverlee, Belgium \\ $\{$ bmd, kostis\}@cs. kuleuven.ac.be
}

\begin{abstract}
The SLG-WAM can be characterized as a sharing approach to implementing tabling: the execution environments of suspended computations are intersperced in the WAM stacks. Stacks are frozen using a set of freeze registers and the WAM trail mechanism is extended so that the suspended computations can be resumed. This technique has a reasonably small execution overhead, but is not easy to implement on top of an existing Prolog system. It is also quite difficult to understand. We propose a new technique for the implementation of tabling: the Copying Approach to Tabling. CAT does not interfere with normal Prolog execution and can be introduced in an existing Prolog system orthogonally. Also, CAT is easier to understand. We have implemented CAT starting from XSB by taking out SLG-WAM and adding CAT. We describe the additions needed for adopting CAT in a WAM implementation. We show a case in which CAT performs arbitrarily worse than SLG-WAM, but on the other hand we present empirical evidence that CAT is competitive and often faster than SLG-WAM. We also briefly discuss issues related to memory management and the impact of the scheduling. - is this true?
\end{abstract}

\section{Introduction}

Tabling in logic programming has been proven useful in a wide range of application areas such as parsing, deductive databases [18], program analysis based on abstract interpretation [5, 3, 12], and recently verification through model checking $[14,4]$. The most practical implementation of tabling is found in XSB [18]: it currently also is the only general Prolog system with tabling. The Table Space of XSB is organized using tries and table access mechanisms are optimised even further through substitution factoring [15]. Also, XSB currently implements two different scheduling strategies described in [10]. In this paper, we will be concerned mainly with the third aspect of tabling, which is the suspension/resumption mechanism and which is orthogonal to the other two. The ability and at the same time need to suspend and resume computations, is a main issue in a tabling implementation, because some subgoals, called generators, generate answers that go into the tables, while other subgoals, called consumers, consume answers from the tables; as soon as a generator depends on a consumer, the generator and the consumer must be able to work in a coroutining fashion, something that is not readily possible in a WAM implementation of Prolog. The execution of the query ?- $\mathrm{p}_{g}(\mathrm{X})$. against the following small program exemplifies this situation.

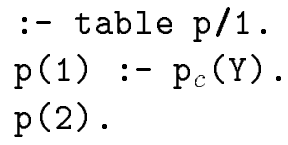


The subscripts $g$ and $c$ denote the occurrence of a subgoal that is a generator or consumer for this particular query. The answer $p(1)$ cannot be generated before $p_{c}$ has consumed the other answer, $\mathrm{p}(2)$, from the answer table that $\mathrm{p}_{g}$ fills. At the moment that $\mathrm{p}_{c}$ consumes the answer $\mathrm{p}(2)$, it must be in an execution state which is the same as when it was selected first. But, in a WAM implementation, backtracking has removed part of that state - because without backtracking, the answer $p(2)$ could not have been generated - so the state of $p_{c}$ must be preserved. The SLG-WAM [17], the abstract machine of XSB, preserves consumer states by freezing them, i.e. by not allowing backtracking to reclaim space on the stacks as is done in WAM. In implementation terms, this means that the SLG-WAM has a extra set of freeze registers, one freeze register for each of heap, trail, local and choice point stack ${ }^{1}$. Moreover, the trail needs to record also values because they have to be reinstalled together with the consumer.

This management of suspension and resumption through freezing and the extended trail mechanism slows down execution which is not related to tabling: this overhead is actually smaller than people usually assume (order of $10 \%$ for an emulated implementation as reported in [17]), but we will show in this paper that it can be completely avoided through the adoption of CAT. Moreover, freezing is a difficult to understand change to WAM and requires a fair amount of careful modifications to some of WAM's instructions. In short, the SLG-WAM machinery is not easy to put into an existing Prolog system and this might be the main reason why logic programming systems do not yet generally offer tabling. CAT solves both problems: it is easier to understand exactly because it allows tabling to be added to an existing WAM-based system without modifying the underlying WAM. One might think that whether CAT is easier to understand than freezing, is a matter of taste. However, our experience written down in [7] indicates differently.

Instead of preserving the consumer state through freezing, one could imagine that the whole state of the abstract machine (i.e. all the stacks) is saved in a separate memory area, and then execution just fails over the consumer. When we need to reinstall the consumer, we can just revert to the saved copy and feed the consumer with its answers. This is not a good solution for two reasons: copying the whole WAM state is unnecessary (as we will show later) and it also leads to unnecessary recomputation. The execution of the query ?- $\mathrm{p}_{g}(\mathrm{X})$. against the following program shows the latter.

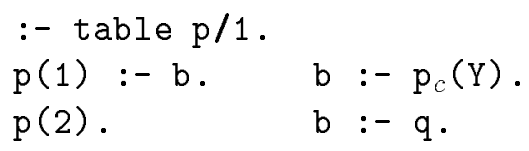

The state of the abstract machine at the moment $p_{c}(Y)$ is called, contains the choice point for $\mathrm{b}$. Still, the second alternative of $\mathrm{b}$ will have been exhausted by the time $\mathrm{p}_{g}(\mathrm{X})$ generates its first answer (in this case $\mathrm{p}(1)$ ). When we reinstall the consumer $\mathrm{p}_{c}(\mathrm{Y})$, we do not want to reinstall the alternative for $\mathrm{b}$ as the goal $q$ represents an arbitrary amount of (re)computation that can - and in the presence of side-effects should - be avoided. Thus, a more selective copying of the WAM state can and should be done. CAT does exactly this: it copies selectively (and incrementally) execution states of consumers and reinstalls these copies when needed.

Unlike the SLG-WAM which modifies some WAM instructions due to trailing and freezing, CAT does not require any changes to the WAM, only additions in the form of a few new instructions which can be alternatively seen as new built-in predicates. These instructions are only generated in clauses belonging to tabled predicates. In this respect, i.e. as far as the tabling instructions are

\footnotetext{
${ }^{1}$ Throughout this paper, we assume a WAM model with environment and choice point stacks separated (as XSB or SICStus Prolog implement) rather than combined as in the original WAM. We also assume that stacks grow downwards, i.e. higher in the stack means older, lower means younger.
} 
concerned, CAT and SLG-WAM only differ in the actions corresponding to these new instructions. The choice points for tabled subgoals in CAT differ slightly from usual WAM choice points but this is not visible for non-tabled execution. Moreover, unlike SLG-WAM, no freeze-registers are needed for CAT, nor the more complicated trail mechanism. In short, CAT allows introduction of tabling into a WAM like implementation without any performance overhead for non-tabled code. Therefore, we believe that CAT is an attractive approach to incorporate tabling in any logic programming system.

In the next section, a brief introduction to tabling and the SLG-WAM is given and some terminology is set. We also introduce a source to source transformation to which SLG-WAM actions are related. The same transformation is later used to relate to CAT. In Section 3, we explain CAT step by step in situations of increasing complexity. All along the additions to WAM are introduced and memory management is discussed. Section 4 discusses a worst case behaviour of CAT and indicates shortly how to deal with it. Section 5 presents in more detail the CAT implementation and the relation between SLG-WAM and CAT. Section 6 compares the performance of CAT and SLG-WAM in the context of XSB. We end with an overview of related and future work.

\section{Tabling and SLG-WAM: Concepts and Terminology}

In this section, we only present concepts and terminology of tabled evaluation and of the SLG-WAM which are necessary to make the paper reasonably self-contained. We assume the usual terminology of logic programming and refer the reader to [2] for issues related to SLG resolution and to [17] for a detailed description of the SLG-WAM. We will mostly restrict ourselves to definite programs; the ability to support well-founded negation in CAT (described briefly in Section 5) is indeed an issue orthogonal to the suspension/resumption mechanism that the underlying abstract machine uses. We keep the presentation informal and refer to [17] for a more formal treatment of the notions that are described below.

\subsection{Basic overview of tabling}

A tabled program is a program augmented (either automatically or by the programmer) with tabling declarations of the form:

$$
\text { :- table } p_{1} / n_{1}, \ldots, p_{k} / n_{k} \text {. }
$$

where $p_{i}$ is a predicate symbol and $n_{i}$ is an integer. These declarations ensure that all queries to the predicate $p_{i}$ of arity $n_{i}$ will be executed using tabled evaluation (e.g. SLG resolution). Other predicates are implicitly assumed as non-tabled in which case SLD resolution is used for queries to these predicates. Slightly abusing terminology, we will speak of tabled subgoals as well as tabled predicates. Following SLG resolution we will consider two tabled subgoals to be the same if they are variants of each other; i.e. identical up to variable renaming; however note that this is also an orthogonal issue to the design of SLG-WAM or CAT. Tabled subgoals which are encountered in the evaluation of a query against a program are persistently stored in a global data structure called a subgoal table. When a tabled subgoal, $s$, is called, a check must be made to see whether $s$ exists in the subgoal table or not. This is the purpose of the SLG NEW SUBGOAL operation. If $s$ is new, it is termed a generator, it is entered in the table and will use PROGRAM CLAUSE RESOLUTION to derive answers. Through the NEW ANSWER operation, the set of derived answers of $s$ will also be recorded in a global data structure called the answer table of $s$. Note that there is a one-toone correspondence between generators and answer tables. If, on the other hand, (a variant of) $s$ 
already exists in the table, it will resolve against answers from its answer table. In this case, we call the subgoal a consumer of $s$. Answers are fed to the consumer one at a time through the ANSWER RETURN operation.

A basic concept in tabled evaluations is completion of (generator) subgoals and their associated answer tables. Informally, a subgoal $s$ (and its answer table) is called complete if all its answers have been derived. On a slightly more operational level, through the SLG COMPLETION operation a subgoal can be determined as complete if all program clause resolution has finished and all instances of this subgoal (the generator and all consumers) have resolved against all derived answers. As there might exist dependencies between subgoals, it is often the case that subgoals cannot be determined complete on an individual basis, but their (mutual) dependencies also have to be taken into account. This suggests that the subgoal dependency graph $D G$ has to be examined and sets of mutually dependent subgoals can be completed when they are involved in a strongly connected component $\Lambda$ of $D G$ that is independent: i.e. none of $\Lambda$ 's subgoals depends on a subgoal outside $\Lambda$. When all subgoals are completed, the evaluation has reached a fixpoint and stops.

\subsection{Implementation of tabling in the SLG-WAM}

As the first example in the introduction shows, tabling cannot be implemented using the pure depthfirst search of the WAM: this is mainly due to the fact that the generation and consumption of answers are asynchronous and interleaved events. This means that an abstract machine for tabling has to maintain or reconstruct execution environments of consumers until these have consumed all answers that are generated for the subgoals; i.e. consumers have to be retained until fixpoint or completion of the associated generators. Likewise, newly derived answers must be queued to resolve against subgoals which do not necessarily correspond to the current execution environment. Also notice that the abstract machine may need to switch back and forth between different consumers multiple times. The following example shows this.

Example 2.1 Consider the execution of the query ?- $\mathrm{p}_{g}(\mathrm{Y})$. which succeeds for all integers between -10 and 10 (included) against the following tabled program:

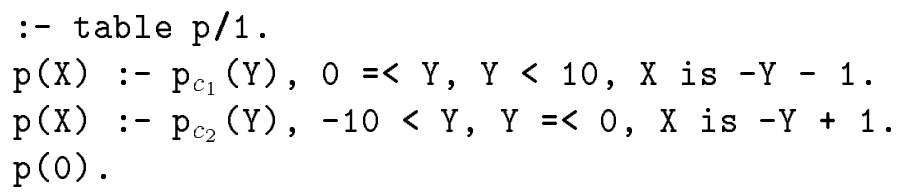

The two consumers that are encountered have been given an index for ease of reference. The abstract machine needs to alternate between consumers $\mathrm{p}_{c_{1}}(\mathrm{Y})$ and $\mathrm{p}_{c_{2}}(\mathrm{Y})$ multiple times before all answers have been generated.

The SLG-WAM offers a particular way to implement all these features which is described below. funny sentence

\subsubsection{Suspending and resuming consumers in the SLG-WAM}

The SLG-WAM implements tabling by suspending consumers when these have exhausted all answers currently in the table and resuming them when new answers have been derived for them. Suspension is performed in SLG-WAM by creating a consumer choice point to represent the suspended environment, freezing all stacks by setting the freeze registers to point to the current top, and then failing to a previous choice point without reclaiming any stack space; in particular, the freeze registers are not reset. Space is not reclaimed above these freeze registers until completion 
of the appropriate generator. Resuming, besides restoring the WAM registers to the values saved in the consumer choice point, uses the addresses and the values saved in a forward trail $[21,20]$ to restore variable bindings along the path to the suspended consumer; see [17] for exactly how this is done. An unconsumed answer is then returned to the restored consumer and execution continues by taking the forward continuation of the restored computation.

In Example 2.1, each of the two consumers trivially needs to be suspended upon its creation as the associated table contains no answers at that moment. The first answer, $p(0)$, is derived using the third clause and let us assume that it is first returned to the generator $\mathrm{p}_{g}(\mathrm{Y})$. For execution to continue after that, one of the two consumers, e.g. $\mathrm{p}_{c_{1}}(\mathrm{Y})$, needs to get resumed to consume the answer $p(0)$. This produces another answer, $p(-1)$, which is also inserted in the table and returned to the generator and to the "current" consumer $\mathrm{p}_{c_{1}}(\mathrm{Y})$. Since no new answers can be generated by doing so, the abstract machine will come at a state where the only remaining action is for the second consumer $\mathrm{p}_{\mathrm{c}_{2}}(\mathrm{Y})$ to get resumed to consume the answers in the table. This produces another two new answers $\mathrm{p}(1)$ and $\mathrm{p}(2)$ which will at some point have to be returned to the first consumer which is now suspended. This process, i.e. switching execution environments between consumers, will continue until all consumers have resolved against all answers.

It can thus be seen that the purpose of freezing is that execution states of consumers are retained until the derivation and consumption of all their answers. Furthermore, note that suspension interacts with completion: if upon consuming the last currently available answer of a subgoal, the subgoal cannot be determined complete, i.e. that it has all its answers, the consumer needs to be suspended so that its execution environment is available if new answers are generated for it. If, on the other hand, a consumer of a complete subgoal is encountered, a completed table optimization can be performed: suspension through freezing is not necessary and the consumer can backtrack through the answers in the table as if they were program clauses stored as facts.

\subsubsection{Scheduling and incremental completion in the SLG-WAM}

Even from the execution trace of the above simple example, one can see that tabling allows an additional level of flexibility in its control. This flexibility is related to when answers are returned to subgoals that consume them. For instance, the abstract machine can postpone the consumption of answer $\mathrm{p}(-1)$ by $\mathrm{p}_{g}(\mathrm{X})$ and $\mathrm{p}_{c_{1}}(\mathrm{Y})$ and choose to return it to $\mathrm{p}_{c_{2}}(\mathrm{Y})$ first. This choice is determined by a scheduling strategy. SLG resolution allows for many different scheduling strategies each of which can have different performance characteristics; see [10]. Naturally, a common requirement for all scheduling strategies is that all answers of a subgoal are returned to all its consumers. XSB currently implements two different scheduling strategies called batched and local scheduling. Both of them are based on partitioning the subgoals encountered during the course of an evaluation into scheduling components. As this partitioning also interacts with and is influenced by completion we examine scheduling and completion together.

In definite programs, completion (determining fixpoint) can be postponed till the end of the evaluation. However, for the SLG-WAM to reclaim space and thus be effective on large programs a more fine grained, incremental completion is needed. To efficiently perform incremental completion, the SLG-WAM (and CAT) contains an area of memory new to the WAM, the Completion Stack. The completion stack can be seen as a restriction of the choice point stack to just the choice points for generators and is used to efficiently keep track of dependencies between subgoals and of scheduling components. Specifically, the completion stack maintains, for each subgoal $s$, a representation of the older (highest) generator subgoal $s_{L}$ upon which $s$ or any subgoal below $s$ may depend. This subgoal is called the leader of its scheduling component. When $s$ and all subgoals 
younger than $s$ have exhausted all program clause resolution, $s$ can be checked for completion. If $s$ is the leader of its component, $A$, and thus does not depend on subgoals higher in the stack than itself and ? if all consumers of subgoals in $A$ have consumed all answers, then $s$ and all other subgoals in $A$ can complete and the (possibly frozen) space that corresponds to subgoals of the component can be reclaimed. Otherwise, if the leader $s_{L}$ is higher in the completion stack than $s$, then $s$ may depend upon subgoals that appear higher than itself on the completion stack, and execution backtracks to the previous alternative without reclaiming any space. This is the main idea behind the implementation of incremental completion in the SLG-WAM (see [17] for more details).

In both batched and local scheduling strategies, scheduling of ANSWER RETURN operations is based on (and limited to the subgoals of) the component that is on the top of the completion stack. This explains why these are called scheduling components. More specifically, the leader of the topmost component is responsible for checking whether all answers have been returned to all consumers of subgoals that it leads, and schedule ANSWER RETURN operations if unresolved answers exist for some consumer. This check, called fixpoint_check in [17], is needed independently of whether each generator schedules its consumers or not after performing all program clause resolution and checking whether it can complete. Scheduling of consumers on failing back to the leader is always possible as some scheduling strategies (including the above two) cannot determine fixpoint in a purely stack-based manner; see $[17,10]$ for why this is so. To appreciate the design of CAT, it is thus important to keep in mind that consumers of subgoals of a scheduling component may need to be resumed when execution has failed back to the leader.

\subsubsection{Overhead of the SLG-WAM}

As already noted, the suspension/resumption mechanism of the SLG-WAM incurs a small but nonnegligible overhead to even non-tabled execution. Before finishing our overview of the SLG-WAM we can now make this overhead more concrete: For example, to place a choice point a comparison between the $\mathrm{B}$ register and the choice point freeze register (BF register) is needed; similarly for trailing or allocating an environment. Checking whether bindings are conditional is also more complicated. Normal execution is also penalised by the introduction of the forward trail: every trailed binding needs more space than in WAM and more time to create it. For an emulated implementation, this overhead is in the order of $10 \%$ and probably not bigger for a native code implementation.

\subsubsection{Tabling explained as a source to source transformation}

The purpose of this section is purely pedagogical: to enhance understanding of the basic actions of the SLG-WAM. We do this by presenting a simple source to source transformation that affects only the tabled predicates. While it is the case that such a source to source transformation hides certain aspects of a tabling implementation, it also illustrates clearly that it is possible to implement tabling by adding a set of (admittedly quite complex) built-in predicates to an existing Prolog implementation. The idea of implementing tabling by a source to source transformation exists already in $[9,22]$ albeit in a completely different flavour. We also note that Mercury release 0.8 [19] has implemented tabling with a source to source transformation which resembles the one below but was however developed independently.

We show the source to source transformation on an example of which the generalisation is straigthforward. Let a tabled predicate foo $/ \mathrm{m}$ be defined as follows: 


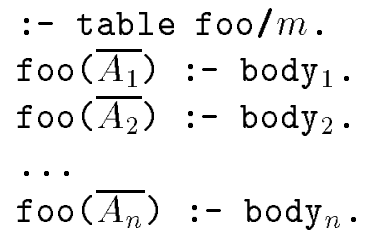

where $\overline{A_{i}}$ denote the particular head arguments of foo/ $m$ and $\bar{X}$ below denotes $m$ new variables, then foo/ $m$ is source transformed to:

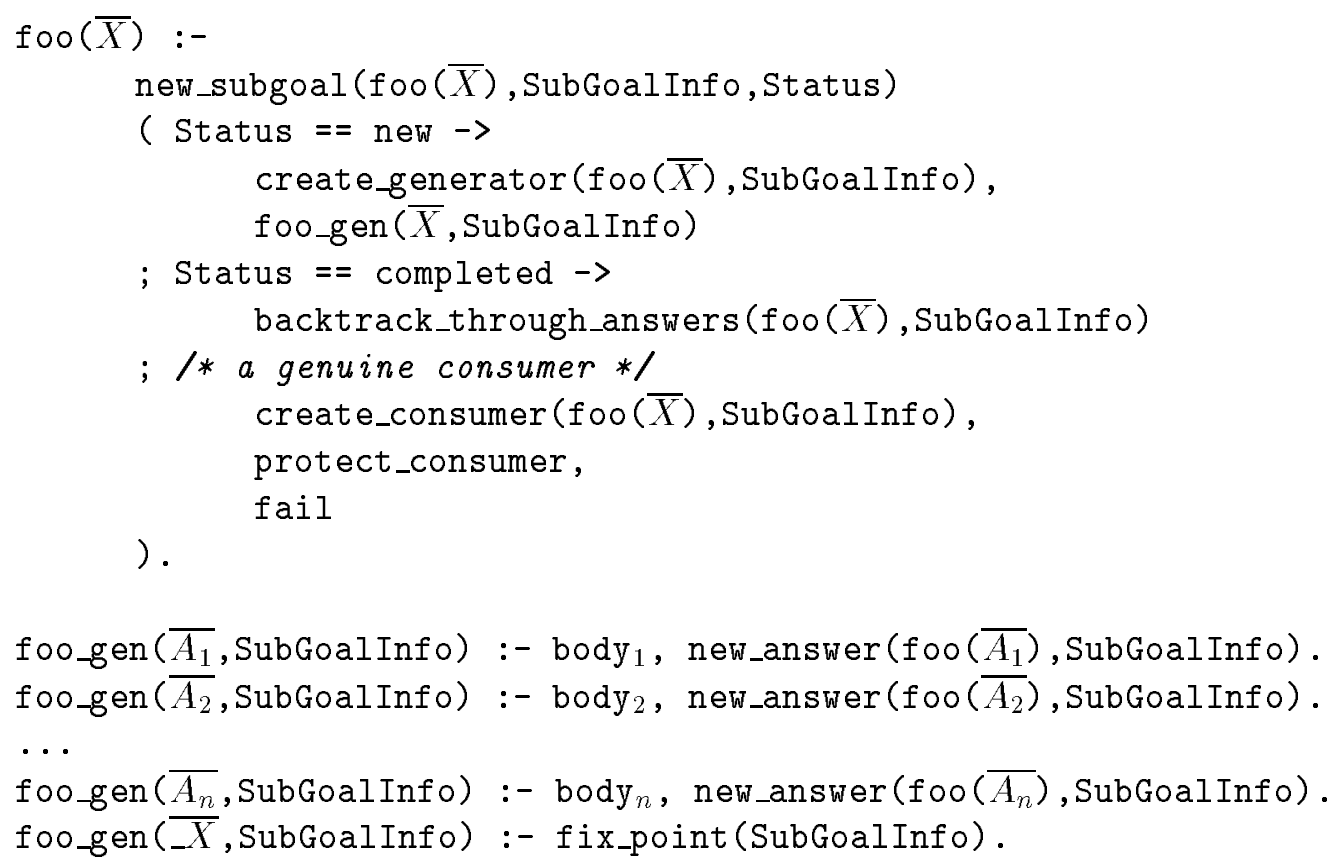

The functionality of the newly introduced built-ins is as follows:

new_subgoal/3 always succeeds and implements the functionality of the NEW SUBGOAL SLG operation (cf. Section 2.1). It finds (or inserts) the subgoal in the table returning a handle to its SubGoalInfo record, and in doing so it also determines its status: whether the subgoal is new to the evaluation or not and whether it is already completed. If the subgoal was just inserted in the table (i.e. it is new), the current subgoal is a generator and the subsequent actions are explained below. If the subgoal is not new to the evaluation, the current subgoal is a consumer and no clauses of the original predicate foo $/ 2$ will be executed. If the corresponding table entry is completed, consumption of answers from the table can be initiated by the built-in backtrack_through_answers/2 which accesses the answer table through its second argument and implements the completed table optimization discussed in Section 2.2.1. Otherwise,

create_consumer/2 installs a consumer choice point, i.e. a data structure that contains a WAM choice point and an extra field, denoted by LastAnswer, which keeps track of which answers from the answer table under construction have been consumed so far by this consumer; the alternative field in this choice point points to an instruction answer_return which implements the functionality of the ANSWER RETURN SLG operation from Section 2.1: it basically does nothing more than consume the next answer if there is any and otherwise fails.

protect_consumer/0 for the SLG-WAM means that the freeze registers are adapted so that back- 
tracking will not destroy the consumer state; as an example, the freeze register $\mathbf{H F}$ for the heap is set to the current top of the heap $\mathbf{H}$. Notice that in the above transformation, after protect_consumer $/ 0$, there comes a fail. This means that alternative branches of the computation are explored before the consumer gets to consume any answer. An alternative is to let the consumer first consume the currently available answers. However, the choice between such alternatives belongs to the scheduling strategy which is an orthogonal issue. Note that the split up of actions between create_consumer/2 and protect_consumer/ 0 is entirely for explanatory reasons : contrast it for example with the actions when a generator is found. ???

create_generator $/ 2$ it was just decided that this invocation of the predicate is a (new) generator and program clause resolution will be used for this subgoal. Built-in create_generator/2 adds the subgoal to the datastructures needed for completion better formulation. Then execution of program clauses happens by calling the foo_gen $/(m+1)$ predicate - defined by $n+1$ clauses if foo/ $m$ is defined by $n$ clauses - which takes the SubGoal Info record as an extra argument for reasons explained below.

new_answer $/ 2$ comes at the end of each clause of the original predicate. If the answer computed by this clause was derived before and already appears in the table new_answer/ 2 fails; if the answer is new, new_answer/ 2 inserts it in the answer table of the particular subgoal and then computation proceeds normally. The second argument to new_answer/2 indirectly contains information about the corresponding answer table; it was put in the generator choice point by create_generator $/ 2$.

The code for fix_point/ 1 is general:

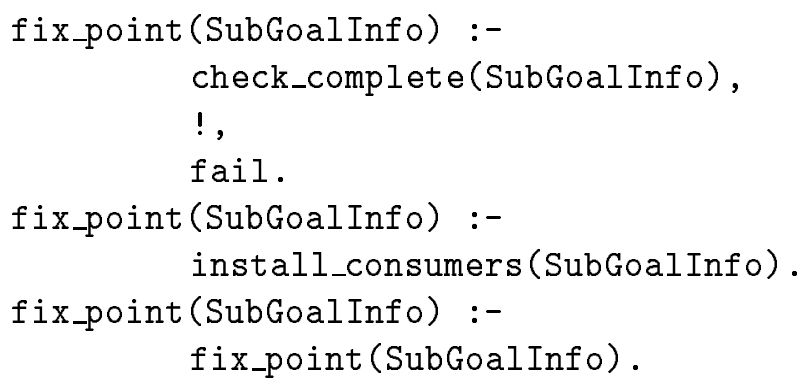

Predicate fix_point/ 1 is called after all program resolution against clauses of a generator is finished. The idea is that $\mathrm{fix}$-point/1 schedules consumers to consume their answers until there are no more of consumers left.

check_complete/1 checks whether the table is complete and all consumers associated with this subgoal have resolved against all answers. Both the table and the consumers are accessible through the SubGoalInfo argument. If the table is complete, the built-in succeeds and the generator is failed back over; the cut-fail combination ensures this. Otherwise, install_consumers/1 gets called; see below. The actions of the check_complete/1 built-in become slightly more complicated when two or more generators depend on each other (for CAT, this situation is dealt with in Section 3.4); see also [17]. It is important to understand that check_complete/1 is only ever executed by generators; the related items in Section 2.1 are the SLG COMPLETION operation and fixpoint_check: the fix point computation is visible in the above code through the recursive call to check_complete/1. 
install_consumers/1 schedules one or more consumers that have not consumed all their answers. In the SLG-WAM, scheduled consumers (whose choice points are still on the stack) are linked to each other and activated by backtracking.

As mentioned, the above description of tabling hides many essential details: how completion is checked for and the data structures that this involves, the handling of negation, the use of the trail when resuming a consumer just to name a few. Also some of SLG-WAM's optimizations are hidden, like for instance substitution factoring [15]. On the other hand, the source transformation gives a good high level description of the actions of the SLG-WAM and it will be instructive to link it later to those of CAT in Section 3.2.

\section{$3 \quad$ A step by step introduction to CAT}

\subsection{A first approximation: no incremental completion}

In this section we make the approximation that incremental completion is not performed: there is only one scheduling component (for all subgoals), and the single leader (which in this approximation runs a monarchy and never changes) does all the scheduling (for all consumers of subgoals that it leads) on failing back to it. In this setting consider how execution goes: At the moment a consumer is found, its consumer choice point is installed on the choice point stack. Figure 1 shows the stacks: generator choice points $G_{0}$ (which is the leader) up to $G_{2}$, followed by the consumer choice point $C$. The vertical dots in between these choice points and above $G_{0}$ denote possible Prolog choice points, not related to execution of tabled predicates. The heap is shown segmented according to the tabling choice points and so is the trail. The same segmentation is not shown in the local stack, as it is a spaghetti stack. From the trail, some pointers point to cells older than the segment between $G_{0}$ and $G_{1}$ : these cells have addresses @1 and @2 in the picture and the values in these cells are $\alpha$ and $\beta$.

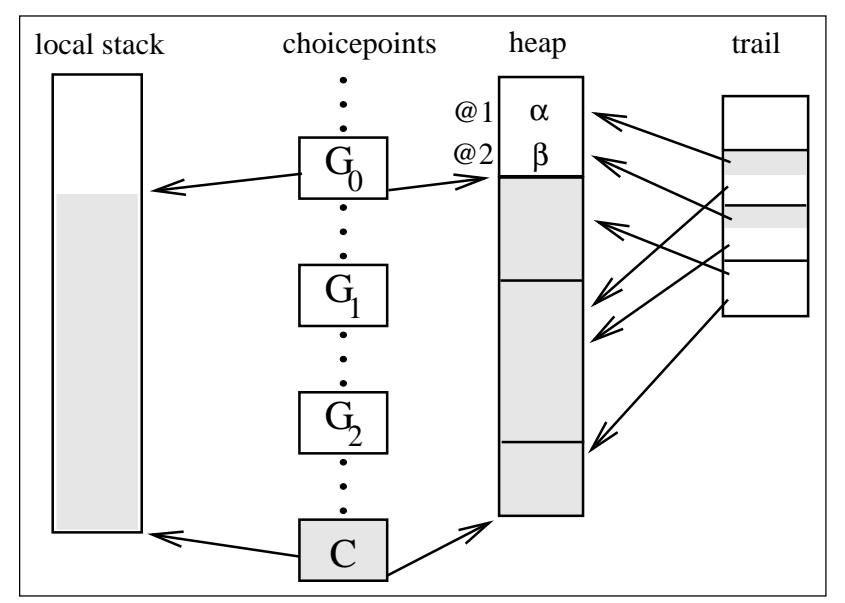

Figure 1: The stacks after the creation of a consumer choice point: the shaded parts indicate what is copied.

Figure 2 shows the information as saved by CAT: there is a (dynamically allocated) frame of fixed size which we name the CAT header. Apart from some bookkeeping fields, it contains a pointer to areas which contain copies of the shaded parts of the stacks: for heap and local stack, 
these shaded areas of Figure 1 are copied as is; they are the part of heap and local stack created between the creation of $G_{0}$ and the consumer $C$. From the choice point stack, CAT only needs to save the consumer choice point: the justification is that at the moment $C$ is scheduled to consume its answers, all the Prolog choice points as well as the non-leader generator choice points will have exhausted their alternatives, and will have become redundant. This also means that when a consumer choice point is reinstalled, this can happen immediately below the leader $G_{0}$.

CAT copies the trail selectively as well: since CAT copies all of the heap between $G_{0}$ and $C$, there is no need to save the trail entries that point into this region and similarly for the trail entries that point to the saved part of the local stack. On the other hand, just saving the trail entries pointing to the older region of the heap (and local stack), is not enough to reinstall the consumer, because backtracking (up to $G_{0}$ ) will have undone the binding of say cell @1 to $\alpha$. It means that CAT must also save the value $\alpha$, or in general the current value of heap (and local stack) cells older than $G_{0}$ and pointed to by trail entries younger than $G_{0}$. Figure 2 shows in more detail a saved trail. We call the CAT header together with the saved stacks, a CAT area.

\section{CAT area}

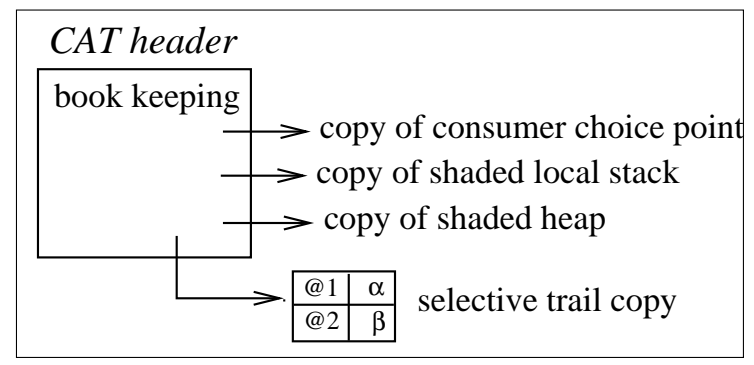

Figure 2: The CAT area showing the selective trail.

After CAT has copied the consumer state, it removes the consumer choice point from the stack and activates a general failure in the WAM. Forward execution might then create other consumers (and CAT areas). Execution will eventually fail back to the leader $G_{0}$.

In this setting, after exhausting all alternatives of $G_{0}$, the leader must also make sure that the consumers consume their answers. The scheduling and restoration of consumers happens as follows: For each consumer, $C$, the saved portion of the heap and the local stack is copied back to its original place. Also, the saved values on the trail are reinstalled and the saved consumer choice point is copied just below $G_{0}$. This reinstalled consumer choice point can now start consuming answers from the tables as in SLG-WAM. After all currently available answers have been consumed, CAT must also update the Last Answer field of the consumer choice point in the corresponding CAT area. Note that after reinstalling the consumer, the trail and choice point stack are in general smaller than at the moment of saving the consumer state. See Figure 3.

In the following sections we will refine CAT, but here already, we have laid out the basics for understanding CAT: the state of a consumer is saved by copying it; this copy consists of the parts of the heap and local stack between the consumer and a generator (which is always older) and similarly for the trail but more selectively; from the choice point stack, CAT only needs the consumer choice point itself.

\subsection{Relating the source to source transformation to CAT}

The built-ins introduced in Section 2.2.4 can now be related to the actions of CAT: 


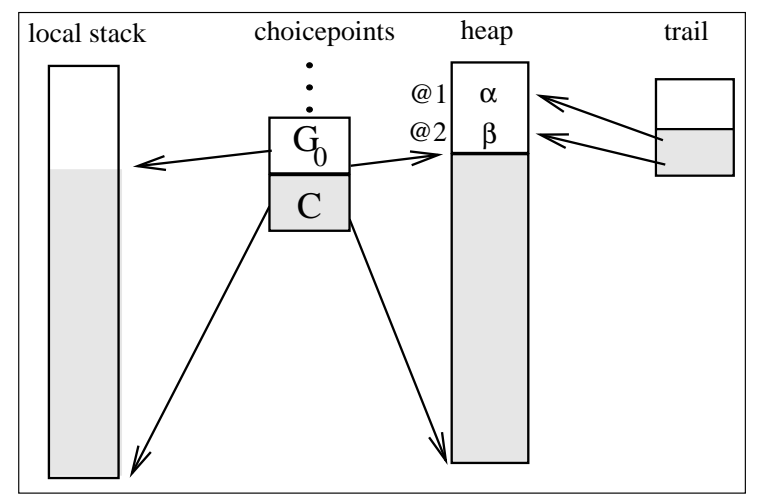

Figure 3: The stacks just after the consumer has been restored.

protect_consumer/0 in CAT means that the consumer state is copied from the stacks to the CAT area: the relevant part of the heap, local stack and (partially) the trail, together with the consumer choice point is saved; also - in contrast to SLG-WAM - the consumer choice point is removed from the stack

install_consumers/1 in CAT schedules one consumer by copying back from the CAT area the saved consumer state; i.e. CAT reinstalls the saved heap and local stack, then reinstalls the bindings from the trail and also copies back the consumer choice point immediately below the generator choice point of the leader. In our prototype CAT implementation we have chosen for scheduling only one consumer at a time, but note that scheduling of a set of consumers is possible as well.

The action of the other built-ins remains the same. At this level of description, the difference between SLG-WAM and CAT seems really small: indeed, CAT can reuse all other actions of the SLG-WAM as well as its optimizations. However, one should keep in mind that CAT does not require changes to the underlying WAM.

\subsection{Adding incremental completion based on fixed leaders}

In the previous section, we assumed that completion was non-incremental and all subgoals belonged to one scheduling component led by a single leader. As mentioned in Section 2.2.2, for definite programs, this is a valid scheme for a tabling abstract machine as scheduling and completion can always be postponed till the end of the evaluation. However, as in the SLG-WAM, in CAT it is more efficient to perform incremental completion and have smaller scheduling components because then:

1. CAT would copy and reinstall smaller parts of the stacks, and

2. subgoals can complete and free the CAT areas of their consumers earlier.

Indeed, look at the execution of ?- $\mathrm{p}_{g}(\mathrm{X})$. against the following program:

$:-$ table $\mathrm{p} / 1, \mathrm{q} / 1$.

$$
\begin{array}{ll}
\mathrm{p}(1):-\mathrm{q}_{g}(\mathrm{Y}) . & \mathrm{q}(3):-\mathrm{q}_{c}(\mathrm{Z}) . \\
\mathrm{p}(2) . & \mathrm{q}(4) .
\end{array}
$$

The answers of $q / 1$ do not depend on the answers of $p / 1$, so a generator of $q / 1$ can be a leader and form a scheduling component in itself. It means that at the moment $\mathrm{q}_{c}(\mathrm{Z})$ is called, its leader 
is not $\mathrm{p}_{g}(\mathrm{X})$, but the subgoal $\mathrm{q}_{g}(\mathrm{Y})$, and the consumer $\mathrm{q}_{c}$ can always be scheduled on failing back to the generator $\mathrm{q}_{g}$. So, in order to reinstall the state of $\mathrm{q}_{c}$, it is enough to copy stacks between $\mathrm{q}_{c}$ and the leader $\mathrm{q}_{g}$. Figure 4 shows a variant of Figure 1: the consumer is $C_{2}$ and its leader is $G_{2}$; the shaded areas to be copied are smaller than before. Note, however, that this schema is not practical because it assumes that the fixed leaders of scheduling components are known beforehand.

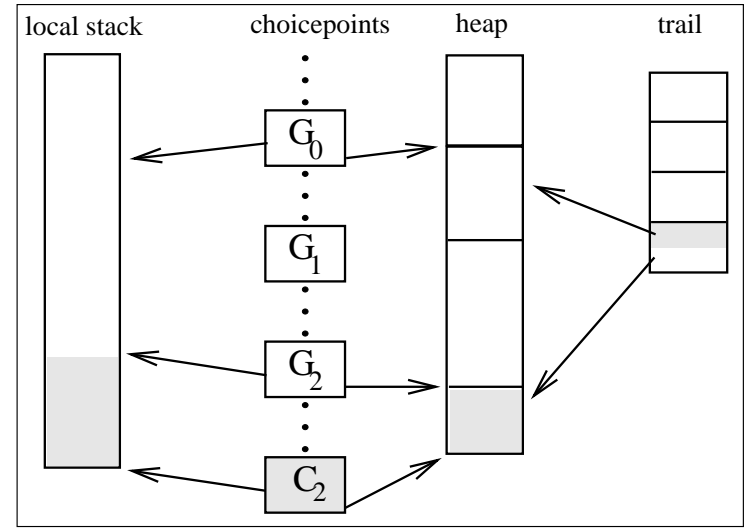

Figure 4: The leader is closer to the consumer: the copy is smaller.

\subsection{A coup: the leader changes}

The principle of the previous two sections was: save consumer state up to the leader generator that might or will schedule the consumer. Even in cases where the fixed leaders are not known in advance, this works well until there is a change of leader: in practice a change of leader happens often. The query ?- $\mathrm{p}_{g}(\mathrm{X})$. executed against the following program shows such a coup:

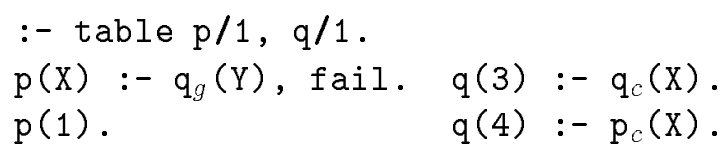

When the consumer $q_{c}$ is saved, its leader (as maintained by dependencies kept in the completion stack) is the generator $\mathrm{q}_{g}$. Indeed, at that point, it is not yet known that the answers of $\mathrm{q}$ will depend on answers of $p$. Later, at the moment the consumer $p_{c}$ is created, a coup takes place: the generator $\mathbf{q}_{g}$ is no longer the leader of a scheduling component and $\mathbf{p}_{g}$ has become the leader of $\mathbf{q}_{c}$. It means that in the future $\mathrm{q}_{c}$ might need to be restored by $\mathrm{p}_{g}$, so the saved state of $\mathrm{q}_{c}$ should at restoration time contain also the part of the stacks between $\mathrm{p}_{g}$ and $\mathrm{q}_{g}$. We could eagerly - at the moment of the coup - save this missing part and add it to the CAT area of $\mathrm{q}_{c}$. The alternative is to wait until execution is about to backtrack over generator $\mathrm{q}_{g}$ : then, CAT saves the increment needed for $\mathrm{q}_{c}$ (i.e. the part of the stacks between $\mathrm{p}_{g}$ and $\mathrm{q}_{g}$ ) and links it up to the CAT area of $\mathrm{q}_{c}$. The advantage of waiting until this moment to save the increment, is that there might be other consumers in the same need for an extension of their CAT area, and thus the part of the stacks between $\mathrm{p}_{g}$ and $\mathrm{q}_{g}$ can potentially be shared between all these consumers, instead of copying the same part for every one of them. This will become more clear below. 


\subsection{Incremental copying of consumer states}

The previous section showed that in the absence of precise information about leaders and scheduling components, there is possibly a need for extending the saved state whenever the leader changes. Now consider the following program, which differs from the one before only by an extra (last) clause for $\mathrm{q} / 1$ :

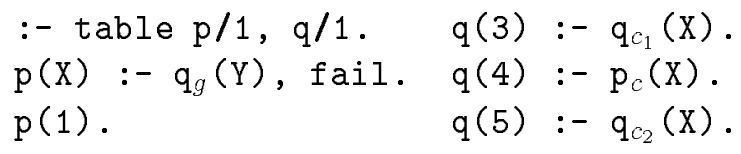

The two consumers for $q / 1$ have been given an index for ease of reference. At the moment consumer $\mathrm{q}_{c_{1}}$ is saved, copying happens up to the leader which is then $\mathrm{q}_{g}$; when consumer $\mathrm{q}_{c_{2}}$ is saved, the coup has happened already, meaning that for $\mathrm{q}_{c_{2}}$, we copy up to $\mathrm{p}_{g}$, the new leader. Later when backtracking happens over the generator $\mathrm{q}_{g}$, we copy the part between $\mathrm{q}_{g}$ and $\mathrm{p}_{g}$ so that this can be linked to consumer $q_{c_{1}}$. Note though that $q_{c_{2}}$ already contains that part of the stacks! So, we have copied twice the same information from the stacks (the part between $\mathrm{p}_{g}$ and $\mathbf{q}_{g}$ ) and it is very difficult to avoid this in the schema which copies a consumer state up to its current leader. Since we have already the mechanism to link parts of saved states, we can use it in a more systematic way as follows: instead of saving a consumer state up to its leader, CAT always saves up to the closest generator $G$. When execution fails back to this generator, all consumers younger than $G$ have copied all information needed for their restoration: they can be scheduled to consume their answers. If $G$ is a leader of a scheduling component, on reaching fixpoint, completion can occur and the space for the CAT areas can be freed. Otherwise, since backtracking over this generator will occur, a new increment up to the previous generator is linked to all the consumers that need it. Applied to the above example, it means that the double copying of the old schema, does not happen anymore.

Figure 5 gives a rough picture of the situation.

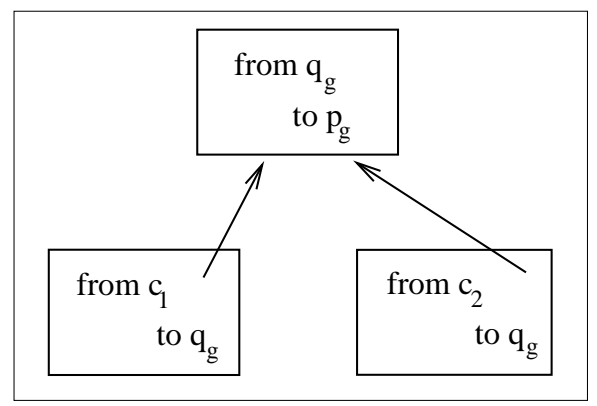

Figure 5: Sharing of CAT areas between consumers. this is a bad picture

This incremental saving of consumer states, is the one finally implemented in CAT: it performs less copying by improving sharing of consumer states. CAT also allows for more flexible scheduling strategies since now even non-leaders can schedule consumers; moreover in the context of CAT, it is natural that a generator can schedule all consumers with a saved state that reaches up to this generator, not just its own. 


\subsection{Managing the CAT areas}

In Section 3.1 we said that the CAT header and the areas in which the consumer state is saved, are allocated dynamically. We indeed rely on the $\mathrm{C}$ library functions malloc() and free(). The interesting point is to see that freeing the allocated areas is quite straightforward: when a leader completes, it means that all the consumers younger than this leader can be discarded. Since a generator has access to the consumers which have their state saved up to this generator, a leader can free easily the CAT areas corresponding to these consumers.

\subsection{Sharing and reusing consumer state}

Consider the execution of ?- $\mathrm{p}_{g}(\mathrm{X})$. against the following example program:

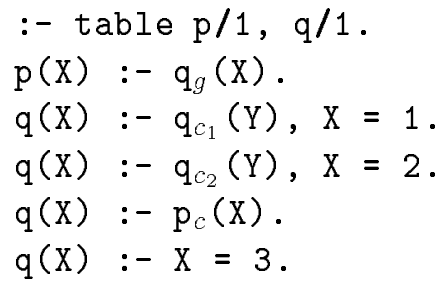

The CAT areas of $\mathrm{q}_{c_{1}}$ and $\mathrm{q}_{c_{2}}$ share the portion of the computation between $\mathrm{p}_{g}$ and $\mathrm{q}_{g}$; let us name this portion $P$. At a certain moment during the execution, $\mathrm{q}_{g}$ has produced the answer $\mathrm{q}(3)$, $\mathrm{q}_{c_{1}}$ has consumed the answer $\mathrm{q}(3)$ and $\mathrm{q}(1)$ already and now it is time to schedule $\mathrm{q}_{\mathrm{c}_{2}}$ : at that moment, $P$ is on the stacks, but with the restoring described earlier, no use of this will be made. It is however not too difficult to take into account during the restoration of $\mathrm{q}_{c_{2}}$ that $P$ doesn't need to be copied back. We have not yet implemented this reuse of restored consumer state. Because of the stack freezing, SLG-WAM gets this reuse more naturally. However, judging from the performance figures, the lack of this reuse in CAT seems not a major problem.

The above raises another issue: due to incremental copying of consumer state, two consumers can share part of their state. However, this sharing is restricted to chunks of the computation between two generator choice points. The analysis in [6] shows that a more fine grained sharing is possible: the increment of saving consumer state, and consequentely the sharing, can be at the level of any choice point. This requires dynamic changes to Prolog choice points, which in spirit resemble [13] (see 7 for more on the latter).

\section{The Problem of CAT}

Before proceeding with more implementation details and the performance evaluation of CAT, we point out a problem with CAT. Consider the program:

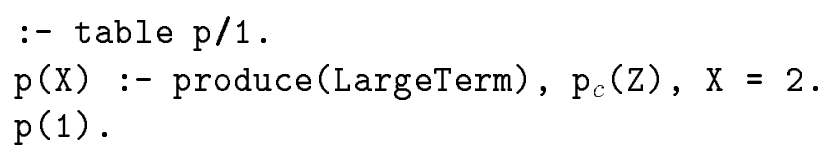

The query ?- $\mathrm{p}_{g}(\mathrm{X})$. produces the answers $\mathrm{p}(1)$ and $\mathrm{p}(2)$ and the consumer $\mathrm{p}_{c}$ is saved and restored once. produce/ 1 is a computation which produces a large term as output argument. In the setting above, the consumer state contains this large term and it is copied once on saving and copied once on restoring the consumer. So the CAT area for a consumer can be arbitrarily large, meaning that both saving and restoring a consumer can take arbitrarily long. The SLG-WAM 
does not suffer from this problem: freezing the stacks is a constant time operation and the cost of restoring a consumer in SLG-WAM is related to the trail. The example shows the problem for the saved heap, but similar examples exist for showing the same effect for the local stack. Since it is easy to construct examples in which consumers have to be restored arbitrarily often, it follows that CAT can be made to perform arbitrarily worse than SLG-WAM. However, this bad behaviour of CAT was not observed in any of the real programs we have so far used tabling for. ${ }^{2}$

As it happens in the above example, this large term is not used in the continuation of the consumer, which means that copying it on saving the consumer was unnecessary. In general one can say that none of the heap entries which are not reachable by the continuation of a consumer, need to be saved. This can be achieved by performing a garbage collection that is restricted to the part of the heap to be copied, just before saving a consumer state. A similar compaction is possible for the local stack. We have explored this idea in more detail in [7]. The results presented there indicate the usual space-time tradeoff for which it is not clear cut what choice is best.

It was mainly the above described behaviour of CAT for which there seemed no completely satisfactory solution that has driven us to design another alternative for implementing suspension/resumption: its name is CHAT and it is descibed in [8] (see also section 7).

\section{Implementation of CAT and Relation to SLG-WAM}

We have implemented CAT within XSB; XSB itself implements SLG-WAM which freezes stacks as a means to save a consumer state. This means that XSB has a more complicated trail, trail test, setting of top-of-stack, etc. As a CAT prefers warmth, we have first heated up XSB, by removing all the freeze related code and replacing it with the plain WAM equivalent. In this warm version of $\mathrm{XSB}$, we have then implemented CAT, reusing from XSB everything related to the implementation of incremental completion and the access and storing mechanisms for tabling of [15] as well as its optimizations. The source to source transformation indicated this possibility.

Besides adding the incremental CAT copying and restoration described in Section 3.5 and slightly modifying the scheduling (as will be explained below), only instructions related to tabling (tabletry, answer_return, ...) which do not belong to the WAM, were changed. So, now there exist two versions of XSB: one that implements SLG-WAM and one that implements CAT. These will form the basis of a comparison between SLG-WAM and CAT. Since to our knowledge, there exists no other implementation of SLG-WAM (nor of CAT) we will henceforth refer to the two mechanisms as if they were implementations.

\section{Current similarities and differences with the SLG-WAM}

In our first ? version of CAT we have retained the incremental completion algorithm of the SLG-WAM. As mentioned, in definite programs, completion can always be postponed and it is mainly used for space reclamation. As the SLG-WAM freezes the stacks at the top, non stackbased selective completion (and space reclamation) of subgoals is not possible without fragmenting the stacks and thus requires stack compaction. As this is probably costly, the SLG-WAM always completes the component in which the youngest generator belongs: therefore, the SLG-WAM uses a completion algorithm based on approximate subgoal dependencies. This algorithm is known to trap subgoals in scheduling components (see [17]) and may arbitrarily postpone their completion despite the fact that they are independent of other subgoals. CAT, as a true feline, is much more flexible in completion because reclamation of the CAT areas is not based on compaction. We plan

\footnotetext{
${ }^{2}$ The bad figures for read_o in Table 1 and 2 have another reason: see Section 6.
} 
to add an exact completion algorithm to our CAT, but for fairness, in this paper we compare CAT and SLG-WAM under the same completion algorithm.

Unlike the SLG-WAM, CAT reclaims the trail and choice point stack even before completion of a component. ${ }^{3}$ In particular, it reclaims generator choice points for non-leaders when these have exhausted program clause resolution. This means that the substitution factor variables (an optimization of [15]) cannot be stored in the choice point stack as in the SLG-WAM, but must be stored in a place that survives backtracking until completion: we have opted for the heap. As another small technical point, since non-leader generators are reclaimed by CAT, local scheduling is implemented by creating a CAT area for generators that are not leaders. This is in accordance with the definition of local evaluation that specifies that these generators behave as consumers (cf. [10]).

Finally, as explained in Section 3.5, CAT allows for more flexible scheduling algorithms, in particular more fine-grained ones. However, the current version only performs scheduling on failing to the leader in a manner similar to the fixpoint_check of the SLG-WAM. A difference with the SLG-WAM is that scheduling decisions are taken more often by CAT since CAT can reinstall only one consumer at a time, while SLG-WAM can schedule several consumers in one go.

\section{Extending CAT to normal logic programs}

\section{needs more about negation ...}

The XSB implementation of the SLG-WAM evaluates programs according to the well-founded semantics. There is really nothing that prevents CAT to also work for this class of programs as the handling of negation is an orthogonal issue. The same low-overhead (around 1\%) mechanisms that the SLG-WAM uses to maintain exact subgoal dependencies [17], to detect and break cycles through negation can be combined with CAT as well. Space limitations prohibit a full discussion but we simply note that the handling of negative (tabled) literals in CAT is analogous to the handling of consumer subgoals: the execution state of negative literals is also preserved in a CAT area by copying. A small difference is that a negation suspension frame [17] rather than a consumer choice point is copied and that this frame is reinstalled once and at the place of the generator choice point (upon its completion) rather than immediately below it.

\section{Performance Evaluation}

As expected, CAT performs better than SLG-WAM in Prolog code; around $10 \%$ according to our measurements (see also [17]). Also, CAT and SLG-WAM have indistinguishable performance in artificial tabling benchmark programs from the database community like transitive closures over chains, cycles and trees of various lengths and same generation over cylinders (cf. $[18,17,10])$. So we compare CAT and the SLG-WAM on more realistic sets of programs from an application area where tabling has been proven worth having in a general purpose logic programming system: abstract interpretation. All measurements were conducted on an Ultra Sparc 2 (168 MHz) under Solaris 2.5.1. Times are reported in seconds, space in KBytes. Space numbers measure the maximum use of the stacks (for SLG-WAM) and the total of max. stack + max. CAT area (for CAT).

\footnotetext{
${ }^{3}$ Actually, it reclaims all stacks, but parts of heap and local stack stay in CAT areas.
} 


\subsection{A benchmark set dominated by tabled execution}

The first benchmark set is taken from [3]: the programs perform type analysis by program abstraction and execution of the abstracted program under tabled evaluation. Tabling is used both for termination, efficient storage of the analysis results and to avoid redundant subcomputations in the domain-dependent abstract operations (see [3]).

With the exception of a few utility predicates like append/3, all other predicates are tabled in this benchmark set and the size of the table space (not shown) is quite large: this set of benchmarks programs is heavily dominated by tabling operations.

Tables 1 and 2 show time and space performance of the analysis under the two scheduling strategies of XSB. On this set of programs, SLG-WAM performs more or less the same time-wise with batched (B) and local (L) strategy with a very noticeable advantage for the local strategy in space consumption as its scheduling components are tighter. CAT under local scheduling performs

\begin{tabular}{||r||c|c|c|c|c|c|c|c||}
\hline & cs_o & cs_r & disj_o & gabriel & kalah_o & peep & pg & read_o \\
\hline \hline SLG-WAM(B) & 0.23 & 0.45 & 0.13 & 0.17 & 0.15 & 0.44 & 0.12 & 0.58 \\
\hline CAT(B) & 0.22 & 0.41 & 0.13 & 0.15 & 0.14 & 0.50 & 0.15 & 0.92 \\
\hline \hline SLG-WAM(L) & 0.23 & 0.43 & 0.13 & 0.16 & 0.16 & 0.42 & 0.12 & 0.61 \\
\hline CAT(L) & 0.22 & 0.42 & 0.12 & 0.15 & 0.14 & 0.40 & 0.11 & 0.55 \\
\hline
\end{tabular}

Table 1: Time performance of CAT vs. SLG-WAM under different scheduling strategies.

slightly better than SLG-WAM in time and slightly worse in space. CAT under batched scheduling is slower than the SLG-WAM for the last three benchmarks by 15-60\%: these are also the benchmarks for which CAT uses more than 10 times more space than SLG-WAM. This behaviour is mostly due to the approximate completion algorithm that is used. In this benchmark set, a high percentage of the tabled subgoals, and more specifically the abstract operations, is semi-det: i.e. produces at most one answer. Because completion is based on an approximation of subgoal dependencies, these semi-det subgoals often get trapped in an approximate scheduling component and cannot be completed on their own. As it is not known whether new answers will be derived for these subgoals, their consumers have to suspend (and create a CAT copy) rather than use the completed table optimisation (see Section 2.2). Some extra measurements for the read_o program shed

\begin{tabular}{||r||c|c|c|c|c|c|c|c||}
\hline & cs_o & cs_r & disj_o & gabriel & kalah_o & peep & pg & read_o \\
\hline \hline SLG-WAM(B) & 9.7 & 11.4 & 8.8 & 20.6 & 40 & 317 & 119 & 512 \\
\hline CAT(B) & 13.6 & 19.4 & 11.7 & 45.3 & 84 & 3836 & 1531 & 5225 \\
\hline \hline SLG-WAM(L) & 6.7 & 7.6 & 5.8 & 17.2 & 13.3 & 19 & 15.8 & 93 \\
\hline CAT(L) & 7.9 & 10.7 & 7.1 & 29.5 & 12.5 & 17 & 23.5 & 246 \\
\hline
\end{tabular}

Table 2: Space performance (KBytes) of CAT vs. SLG-WAM under different scheduling strategies.

more light: under batched scheduling 3112 consumers are saved (max. CAT area of 5208 KBytes) and only 192 times a consumer is restored (totaling 371 KBytes) to consume new answers; in contrast, the corresponding numbers for local scheduling are 264 consumers saved and 224 restorations (240 and 371 Kbytes respectively - the bigger restoration is due to sharing of the saved space by incremental copying, but not by restoration: see Section 3.7 ). With the current approximate completion algorithm, under batched scheduling, CAT performs worse than SLG-WAM. We strongly 
believe that for this benchmark set CAT will be more competitive to SLG-WAM (under batched scheduling) if completion is based on exact subgoal dependencies (cf. also Section 5).

\subsection{A more realistic mix of tabled and Prolog execution}

Our experience is that tabling is used selectively and is thus usually only one component in realistic programs: in the case of abstract interpretation for instance, not all analyses benefit from caching the abstract operations as this depends on whether there is redundancy in the computation. Such is the case on the following set of benchmarks from [12]. The abstract operations are implemented in Prolog and take a high percentage of the total execution time (around 75-80\%). The programs perform program analysis based on a complex abstract domain which is able to capture modes, linearity, freeness and sharing. As, contrary to the analysis of the previous section, this analysis is based on an abstract least upper bound operation, i.e. keeping only the lub and deleting all other answers, local scheduling performs much better (there is an order of magnitude difference; see also [10]) than the batched strategy in this benchmark set. Regardless of the issue SLG-WAM vs. CAT, only the local scheduling makes sense in this program set.

Tables 3 and 4 compare SLG-WAM and CAT in time and space. Overall, CAT performs timewise slightly better (5-20\%) in this set. It performs considerably better (25-140\%) than SLG-WAM in space with read as the only exception.

\begin{tabular}{||r||c|c|c|c|c|c|c|c|c|c|c||}
\hline & akl & color & bid & deriv & read & browse & serial & rdtok & boyer & plan & peep \\
\hline \hline SLG-WAM & 1.48 & 0.67 & 1.11 & 2.56 & 9.64 & 32.6 & 1.17 & 3.07 & 10.02 & 7.61 & 9.01 \\
\hline CAT & 1.24 & 0.62 & 0.97 & 2.50 & 9.56 & 32.2 & 0.83 & 2.75 & 9.96 & 6.38 & 8.54 \\
\hline
\end{tabular}

Table 3: Time performance of CAT vs. SLG-WAM.

\begin{tabular}{||r||c|c|c|c|c|c|c|c|c|c|c||}
\hline & akl & color & bid & deriv & read & browse & serial & rdtok & boyer & plan & peep \\
\hline \hline SLG-WAM & 998 & 516 & 530 & 472 & 5186 & 9517 & 279 & 1131 & 2050 & 1456 & 1784 \\
\hline CAT & 552 & 223 & 206 & 486 & 8302 & 7847 & 227 & 821 & 1409 & 1168 & 1373 \\
\hline
\end{tabular}

Table 4: Space performance (in KBytes) of CAT vs. SLG-WAM.

\section{Related Work}

Copying as an alternative to sharing, exists in many places. We just mention a few really closely related systems or techniques.

rearrange

rewritten

First of all, there is an analogy between the SRI-model [20] for implementing OR-parallelism and MUSE [1] on one hand, and the SLG-WAM for implementing tabling and CAT on the other: like the SLG-WAM, the SRI-model has a complicated management of the stacks and switching from one worker to another - the analogue of suspending one consumer to resume another one uses a trail structure that is more complicated than the WAM trail, because bindings have to be undone as well as reinstalled. Like MUSE, CAT avoids complicated stacks by copying the portion of the stacks that is particular to a consumer or in the case of MUSE a worker. We believe this 
analogy is so strong, that although not conscious at the time, we must have been influenced by our knowledge of MUSE when getting the idea for CAT. [1] notes that the overhead of copying is small compared to all other work to be performed and our experience with CAT is similar.

[13] provides a set of library functions for introducing backtracking in $\mathrm{C}$ programs: the parts of the C-stack that must become active again after failure are copied incrementally. This is achieved by changing at run time the return address in activation records. In contrast, in CAT the zones to be copied are delimited by invocations of tabled predicates, so that by compiling these with special instructions, such a change is performed effectively at compile time.

The similarity between the method in [13] and CAT is even more striking, considering that while [13] reduces the complexity of the Prolog spaghetti stack (environments and choice points) by copying and the C-stack (which has a strict stack regime), CAT reduces the SLG-WAM double spaghetti stack (environments, choice points, generators and consumers) to the Prolog spaghetti stack with copying.

A copying technique similar to CAT is used in the abstract interpretation framework AMAI [11]: AMAI is an abstract machine for abstract interpretation whose design was inspired by the SLG-WAM. In order to obtain a fixpoint of the analysis, the continuation of some program points needs to be reanalysed: this can be seen as suspended computation that is later resumed. AMAI achieves this by making a copy of the current state of a recursively analysed goal, including its continuation. The consumer state is saved incrementally in a similar way as in CAT. AMAI is currently implemented in Prolog as an interpreter for the abstract machine. The main difference between AMAI and CAT is that CAT tries to share incrementally saved states between consumers while AMAI attempts no such sharing. Another difference is that CAT inherits from XSB, that dependencies are detected dynamically while AMAI approximates the dependencies statically: this makes sense in abstract interpretation. As a consequence of the static approximation of SCCs, AMAI could also have saved the state of consumers while descending from a generator to a consumer instead of going up again, and in this way sharing of parts of saved states would come even natural.

As mentioned before in Section 4, we have also worked on yet another alternative to freezing for implementing suspension/resumption: the results can be found in [8]. One interesting outcome is that CAT and SLG-WAM offer two extremes in a wide spectrum of possible implementations of suspension/resumption: while the SLG-WAM is extreme in its sharing of state, CAT is extreme in its copying of state. In [8], we describe a hybrid method - CHAT - which has even better implementation properties than CAT as it combines the advantages of CAT copying with the advantages of SLG-WAM freezing, albeit without using freeze registers. This does by no means imply that CAT should be forgotten: it remains the easiest way to understand suspension/resumption of consumers and it offers a very attractive way to implement suspension/resumption of consumers if particular restrictions of the implementation make a hybrid method less convenient. Mercury release 0.8 ([19]) serves as an example: it incorporates tabling using a copying technique, while the hybrid method described in [8] would have been more difficult to adopt because the concept of a WAM choice point does not quite match the non-det stack frame of Mercury. Mercury's copying technique differs from CAT mainly in that copying is less selective. The copying technique employed by Mercury and CAT were developed independently, but they have a common root in a meeting ${ }^{4}$ of the authors of the current paper and Zoltan Somogyi. During this meeting the idea of copying consumer state for a zero-cost-if-not-used implementation of suspension/resumption of consumers came up.

\footnotetext{
${ }^{4}$ September 1996, Leuven
} 
We believe CAT can be useful elsewhere. E.g. in [16] a design for combining tabling and orparallelism is presented. The implementation of tabling in that system is based on the SLG-WAMwhich was at that time the only WAM-based model of implementing tabling, while or-parallelism is supported through environment copying as in the MUSE model. In view of the above mentioned similarity between CAT and MUSE, we believe that CAT also offers a more natural way of combining tabling and or-parallelism as the same basic machinery can be used for satisfying the implementation requirements of both forms of suspend/resume mechanisms.

Since the development and implementation of CAT, we have used CAT to understand better the memory management issues in SLG-WAM: indeed we had been trying before to build a garbage collector for XSB and the lack of understanding of reachability prevented its implementation. Only after developing CAT and using it to understand SLG-WAM, have we been able to design a garbage collector for XSB. For more details see [7].

the following is tentative Finally, in this context it is also worth mentioning the totally different approach to tabling described in [22]: it is entirely based on program transformation and does not require new built-ins as we introduced in Section 2.2.4. A complete comparison between [22] and the SLG-WAM does not exist - unless I find it in his PhD ..

\section{left out future work completely - some is in the conclusion}

\section{Conclusion}

At the offset, this research had one particular aim: design a suspension/resumption mechanism that is orthogonal to the underlying machine into which tabling is build, be it WAM or another machine. With such a mechanism, it would be more attractive for existing LP systems, to incorporate memoization. CAT offers such a mechanism and we showed that CAT is a true alternative to SLG-WAM for implementing the control of tabling: CAT does not impose any overhead on non-tabled execution in a general purpose logic programming system based on WAM. This is good because a Prolog program that employs tabling as an optimisation technique, can still run at the full speed of the underlying Prolog machine for other parts of the computation. CAT can be introduced orthogonally to an existing WAM-like implementation and the idea should be easy to adapt to other LP systems. Therefore, CAT makes it more attractive for existing LP implementations to incorporate tabling: the basic speed of these systems is then not corrupted by introducing tabling. Mercury release 0.8 provides evidence that this is true.

CAT implements only the control of tabling, but the other two components - the tabling data structures and the scheduler - were always orthogonal issues and do not affect the underlying Prolog implementation at all.

CAT has additional advantages: one of them is the understanding of the complicated SLG-WAM machine. SLG-WAM is in some respects the perfect machine for suspension/resumption: suspension is constant time, resumption depends only on the reinstallation of bindings, i.e. the trail. But SLG-WAM is quite difficult to understand exactly because of these properties. CAT provides a more gentle introduction to the issues involved in suspension/resumption.

In the same spirit, CAT proved invaluable in the understanding of the memory management issues when computations are suspended and later resumed. CAT made it possible to finish a much 
earlier started garbage collector for XSB.

CAT allows for more flexible scheduling strategies, but this issue is still to be explored. more to say?

Contrary to what could have been expected from the worst case for CAT, empirical tests show that most of the benchmark programs are not slowed down by the CAT technique compared to implementation of SLG-WAM in XSB. Under the local evaluation strategy, the memory consumption is even most often better than under SLG-WAM.

However, CAT is not the only solution to the initial problem - an implementation of suspension/resumption orthogonal to WAM - as we have explored also a hybrid copying schema - CHAT - described in [8]: depending on considerations related to the underlying machine, either choice of CAT or CHAT is reasonable.

Neither is CAT (or its successor CHAT) a final answer to incorporating tabling in an existing system: indeed, there remains the complex task of designing and implementing a set of primitives that can be hooked up to an existing implementation like a C-library ... see the idea I want to convey?

\section{Acknowledgements}

We are grateful for discussions on the XSB-mailing list; in particular we would like to thank David S. Warren, Terrance Swift and Prasad Rao. should we do this ??? The second author was supported by a K.U. Leuven junior scientist fellowship.

\section{References}

[1] K. A. M. Ali and R. Karlsson. The Muse approach to OR-parallel Prolog. International Journal of Parallel Programming, 19(2):129-162, Apr. 1990.

[2] W. Chen and D. S. Warren. Tabled Evaluation with Delaying for General Logic Programs. Journal of the ACM, 43(1):20-74, Jan. 1996.

[3] M. Codish, B. Demoen, and K. Sagonas. Semantics-Based Program Analysis for Logic-Based Languages using XSB. Springer International Journal of Software Tools for Technology Transfer, Oct. 1998. To appear.

[4] B. Cui, Y. Dong, X. Du, K. N. Kumar, C. R. Ramakrishnan, I. V. Ramakrishnan, S. A. Smolka, and D. S. Warren. Logic Programming and Model Checking. In C. Palamidessi, H. Glaser, and K. Meinke, editors, Principles of Declarative Programming, 10th International Symposium, PLILP'98, Held Jointly with the 6th International Conference, ALP'98, number 1490 in LNCS, pages 1-20, Pisa, Italy, Sept. 1998. Springer.

[5] S. Dawson, C. R. Ramakrishnan, and D. S. Warren. Practical Program Analysis Using General Purpose Logic Programming Systems - A Case Study. In Proceedings of the ACM SIGPLAN Conference on Programming Language Design and Implementation, pages 117-126, Philadelphia, Pennsylvania, May 1996. ACM Press.

[6] B. Demoen and K. Sagonas. CHAT is $\Theta$ (SLG-WAM). Technical Report CW 269, K.U. Leuven, July 1998. Submitted for publication. 